ophthalmological point of view (already for a long time). For one-eyed athletes or sports persons with monocular defective vision protective sports goggles should be obligatory in order to protect the remaining healthy eye.

\section{HOW CAN WE PROTECT ATHLETES FROM DENTAL EROSION?}

${ }^{1}$ Hesham Matabdin, ${ }^{1}$ Paul Ashley, ${ }^{2}$ Pete Wilde, ${ }^{1}$ Ian Needleman. ${ }^{1}$ University College of London, Eastman Dental Institute, London, UK; ${ }^{2}$ Food structure, colloids and digestion, Quadram Institute, Norwich, UK

\subsection{6/bjsports-2021-IOC.315}

Background Our data on more than 800 participants shows that dental erosion (DE) affects up to $45 \%$ of elite athletes and may have physical and psychosocial impacts. The condition is associated with consumption of sports drinks and is exacerbated by reduced salivary flow. The protein rich layer called dental pellicle (DP) may have a protective function for $\mathrm{DE}$ and this might prove to be a useful therapeutic target in elite sport.

Objectives To assess the protective function of DP against DE. Design In vitro study.

Setting Laboratory.

Participants 40 bovine incisors

Interventions Two levels. 1: We exposed twenty bovine incisors to an energy drink ( $\mathrm{pH} 3.1)$ at 24,48 and 72 hours to create an in vitro erosion model. 2: We used fresh human saliva to form dental pellicle on the buccal surface of twenty incisors to create an in-vitro pellicle model. Erosive lesions were investigated both in the presence and absence of a layer of dental pellicle.

Main outcome measurement Mean depth of erosive lesions by optical coherence tomography (OCT) and X-ray Microtomography (XMT).

Results OCT showed the mean thickness of amorphous enamel before immersion in the erosive medium was $64 \mu \mathrm{m}$ (SD 2.3, IQR 54.7 - 63.2). The mean depth of the erosive lesion after immersion was $240.8 \mu \mathrm{m}$ (SD 32.7, IQR 244.6 - 263.8) $(p<0.001)$ at 72 hours. In contrast, mean depth of lesion in the presence of the dental pellicle was 84.3 (SD 18.9, IQR 93.2 - 75.3) $(p<0.03)$. XMT showed the mean depth of erosive lesions was $130 \mu \mathrm{m}$ (SD 28.2), whereas in the presence of DP was $82.4 \mu \mathrm{m}$ (SD 15.6) $(p<0.001)$.

Conclusions Erosion was reduced but not prevented by presence of dental pellicle. This model shows promise as a method to investigate novel interventions to prevent $\mathrm{DE}$ in elite athletes.

\section{MICROBIOME ANALYSIS IN ELITE SPORT}

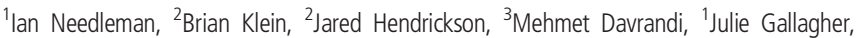
${ }^{1}$ Paul Ashley, ${ }^{3}$ David Spratt. 'UCL Eastman Dental Institute, Centre for Oral Health and Performance, London, UK; ${ }^{2}$ Vedanta Biosciences, Cambridge, USA; ${ }^{3}$ UCL Eastman Dental Institute, Microbial Diseases, London, UK

\subsection{6/bjsports-2021-IOC.316}

Background There is increasing interest in the microbiome in performance and prevention of illness.

Objective To investigate characteristics of oral and gut microbiomes in elite sport.
Design Metagenomic sequencing performed on stool and saliva samples at baseline and three months. Taxanomic identification of the DNA sequence data generated on the Illumina sequencing platform, followed by unsupervised Principle Component Analyses (PCA). UCL Research Ethics Committee ID Number: 6388/002.

Setting 1: A GB Olympic team, $\mathrm{n}=18 ; 2$ : English premiership rubgy team, $\mathrm{n}=18 ; 3$ : Healthy volunteers (non-athletes) $\mathrm{n}=28$. Participants Aged $\geq 18$ years, able to understand consent process, for health controls body mass index18 to $30 \mathrm{~kg} / \mathrm{m} 2$.

Assessment Of Risk Factors Oral health, BMI, use of antibiotics.

Outcome Measurements Unsupervised PCA.

Results Two distinct clusters emerged, one of athletes and one of non-athletes. Specific species-level signatures distiguishing the two clusters as well as each cohort were identified, including Fusicatenibacter sacchrivorans enriched in athlete samples relative to non-athlete controls, Slackia isoflavoniconvertans enriched in rugby relative to both Olympic athletes and nonathletes, and Klebsiella pnemoniae enriched in Olympic cohort relative to both rubgy and non-athletes (all $\mathrm{P}<0.005$ ). For saliva PCA analyses, no distinct clusters emerged between the two athlete cohorts or timepoints. However, specific specieslevel signatures distiguishing the groups were indentified, including multiple Neisseria spp. being elevated in rubgy relative to Olympic athetes at both timepoints, Prevotella histicola being elevated in Olympic athletes relative to rubgy at both timepoints, and Bifidobacterium longum being almost exclusively detected in the Olympic cohort but not rugby.

Conclusions Marked differences in microbiome signatures were found both between elite athletes and non-athletes and between team and individual sport cohorts. Further studies may help identify microbial factors related to optimal food conversion, performance or recovery, and prediction of illness risk.

\section{EQUIPMENT-RELATED RISK FACTORS FOR ACL INJURY AMONG RECREATIONAL SKIERS - A CASE CONTROL STUDY}

${ }^{1}$ Gerhard Ruedl, ${ }^{1,2}$ Markus Posch, ${ }^{2}$ Katja Tecklenburg, ${ }^{1}$ Martin Faulhaber ${ }^{1}$ Martin Burtscher. 'Department of Sport Science, University of Innsbruck, Innsbruck, Austria; ${ }^{2}$ Medalp sportclinic, Imst, Austria

\subsection{6/bjsports-2021-IOC.317}

Background In recreational skiing, an ACL injury is one of the most common diagnosis.

Objective To compare equipment-related risk factors between ACL injured and uninjured skiers.

Design Case-Control study over the 3 winter seasons 2016/ 17-2018/19 in one major Austrian ski resort.

Participants 248 ACL injured (52\% females) and 1054 uninjured skiers (48\% females).

Assessment of Risk Factors ACL injury was diagnosed by MRI in a sports clinic located in the ski area. Ski length [m] and side cut radius $[\mathrm{m}]$ were taken from the information given on the ski and ski length was relativized to body height [\%]. Combined height of ski and binding plate $[\mathrm{mm}]$ at the front $\left(\mathrm{H}_{\mathrm{F}}\right)$ and back part $\left(\mathrm{H}_{\mathrm{B}}\right)$ of the ski binding, and height at the toe $\left(\mathrm{H}_{\mathrm{T}}\right)$ and heel piece $\left(\mathrm{H}_{\mathrm{H}}\right)$ of the ski boot $[\mathrm{mm}]$ were measured with a digital sliding caliper. Quotients between $\mathrm{H}_{\mathrm{F}}$ and $\mathrm{H}_{\mathrm{B}}$ as well as between $\mathrm{H}_{\mathrm{T}}$ and $\mathrm{H}_{\mathrm{H}}$ were calculated. 